\title{
The effect of loading direction on the dwell fatigue properties of Ti-6Al-4V forged bar with highly oriented texture
}

\author{
Kenichi Mori ${ }^{\mathrm{a}, *}$, Shohtaroh Hashimoto ${ }^{\mathrm{a}}$, Toshiyuki Okui ${ }^{\mathrm{a}}$, Yoshihisa Shirai ${ }^{\mathrm{a}}$, Mitsuo Miyahara ${ }^{\mathrm{a}}$ \\ ${ }^{a}$ Nippon Steel Corporation, 20-1 Shintomi, Futtsu, Chiba, 293-8511, Japan \\ *mori.x8f.kenichi@jp.nipponsteel.com
}

\begin{abstract}
$\underline{\text { Abstract }}$
The purpose of this study was to improve our understanding of the effect of macro/micro texture on dwell fatigue properties. The Ti-6Al-4V forged bar with highly oriented texture and fine equiaxed microstructure was used. Dwell fatigue, cyclic fatigue and creep tests were conducted at room temperature by using specimens taken in the longitudinal $(\mathrm{L})$ direction and the transverse $(\mathrm{T})$ direction of the bar. The effects of loading direction on dwell fatigue life and fracture surface morphology were examined in detail. The dwell fatigue life in $\mathrm{T}$ direction was shorter than that in $\mathrm{L}$ direction. The fracture surface morphologies were characteristically varied by loading waveform, amplitude and directions. In the range of $93-95 \%$ of $0.2 \% \mathrm{PS}$, the characteristic large facets were observed in the T direction in dwell fatigue. Detailed analyses revealed that the large facet consists of multiple initiation facets and propagation facets. The propagation facet plane and crack propagation direction appeared to correspond to alpha $(0001)<10-10>$. Furthermore, the relationship between strain rate and life time was compared to that for room temperature creep. The deviation from the Monkman-Grant relationship corresponded to the change of the fracture surface morphology.
\end{abstract}

\section{Introduction}

In titanium alloys used for fan disks of aircraft engines, it is known that the fatigue life significantly decreases by dwell fatigue at about room temperature to $200^{\circ} \mathrm{C}$. Many studies have been conducted on the influence of microstructure, texture or MTR (Micro Texture Region) on crack initiation sites and characteristic facet formation in dwell fatigue [1,2]. Evans et al. described the facet formation mechanism using stress redistribution model and modified Stroh model [3]. Hasija et al. performed analysis accounting for plastic anisotropy and time-dependent plasticity, showed that local stress concentration generated by load shedding mechanism when grains with different orientations are adjacent, and mentioned that the stress concentration could cause the crack initiation [4]. Pilchak et al. discussed characteristic large facets in detail by classifying them into initiation facets and propagation facets [5]. Furthermore, Pilchak et al. showed that the crack growth rate was faster in facet forming than in striation forming [6]. Also, Lang revealed that time-dependent crack propagation occurred during stress retention [7]. Thus, in dwell fatigue, the interaction between fatigue and creep complicates the change in damage mechanism due to macro-/micro-texture and stress conditions. Therefore, in the industrial aspect, it is required to grasp the influence of various factors on dwell fatigue damage and quantitatively predict the fatigue life.

We have been tried to increase the knowledge about the influence of the anisotropy of the texture and the stress condition on the dwell fatigue properties $[8,9,10]$. In this study, the Ti-6Al-4V forged round bar having fine equiaxed microstructures with highly oriented texture was used. Then, the load was applied in the axial or radial direction of the bar, and the fatigue behaviors were investigated. In addition, fracture surface morphologies and damage behaviors were compared with cyclic fatigue and room temperature creep.

\section{$\underline{\text { 2. Experimental Procedures }}$}

The material used in this study was Ti-6Al-4V (Ti-6.3Al-4.0V-0.17Fe-0.18O (mass\%)) forged round bar. The bar has fine equiaxed microstructures with grain diameter of about 10 to $20 \mu \mathrm{m}$ and has texture in which (10-10) ${ }_{\alpha}$ is accumulated in the axial direction of the bar (Figure 1). The specimens were taken in the axial direction (L direction) and the radial direction (T direction) of the bar. The $0.2 \%$ proof stress measured at the strain rate of $8.3 \times 10^{-5} \mathrm{~s}^{-1}$ using specimens with parallel portion of $\varphi 5 \times 30 \mathrm{~mm}$ and gauge length of $25 \mathrm{~mm}$ was $867 \mathrm{MPa}$ in the L direction and $913 \mathrm{MPa}$ in the T direction. The specimens with parallel portion of $\varphi 5.08 \times 15.24 \mathrm{~mm}$ and gauge length of $12 \mathrm{~mm}$ were used for fatigue tests. The fatigue tests were carried out under stress control with the stress ratio of 0.05 , where the maximum stresses were 825 to $870 \mathrm{MPa}$ for L direction and 850 to $915 \mathrm{MPa}$ for $\mathrm{T}$ direction. As for the stress waveforms, cyclic fatigue tests were performed at $1 \mathrm{~s}$ loading / $1 \mathrm{~s}$ unloading cycles, and dwell fatigue tests were performed at $1 \mathrm{~s}$ loading / 120s holding / 1s unloading cycles. The creep tests were carried out with the stress 870 to 905 $\mathrm{MPa}$ for L direction and 905 to $915 \mathrm{MPa}$ for T direction using the specimens with parallel portion of $\varphi 6 \times 30 \mathrm{~mm}$ and gauge length of $30 \mathrm{~mm}$. All mechanical tests were performed at room temperature. Microscopic examinations were carried out using scanning electron microscopes (SEMs), electron back scattered diffraction (EBSD) system. JOEL IT-300 was used for the 
observations of fracture surface morphology. JEOL JSM-7001F, EDAX EBSD camera and OIM software were used for the analysis of the crystal orientation for fracture surface and cross section of the specimens.

(a)
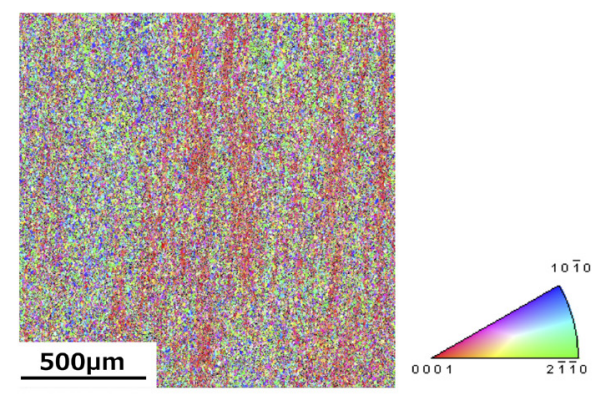

$10 \overline{10}$
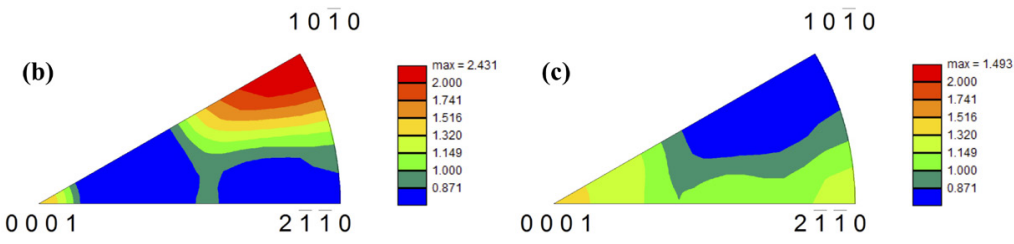

Figure 1. (a) Inverse pole figure map for longitudinal cross section of alpha phase of the Ti-6Al-4V forged bar. Inverse pole figures of the orientation distribution function with respect to the loading direction for (b) $\mathrm{L}$ direction and (c) $\mathrm{T}$ direction.

\section{Results and Discussions}

(1) Fracture surface analysis

Figure 2 shows the relationships between the ratio of maximum stress to $0.2 \%$ proof stress, stress ratio ( $\%$ ), and cycles to failure. In cyclic fatigue, regardless of the loading direction, the relationships between fatigue life and stress ratio are almost the same. On the other hand, in dwell fatigue, the relationships are obviously different according to the loading direction, and the fatigue lives for $\mathrm{T}$ direction significantly decreased. When the stress ratio is $95 \%$, the dwell debit is suppressed to 2.1 for L direction, but high at 8.6 for $\mathrm{T}$ direction.

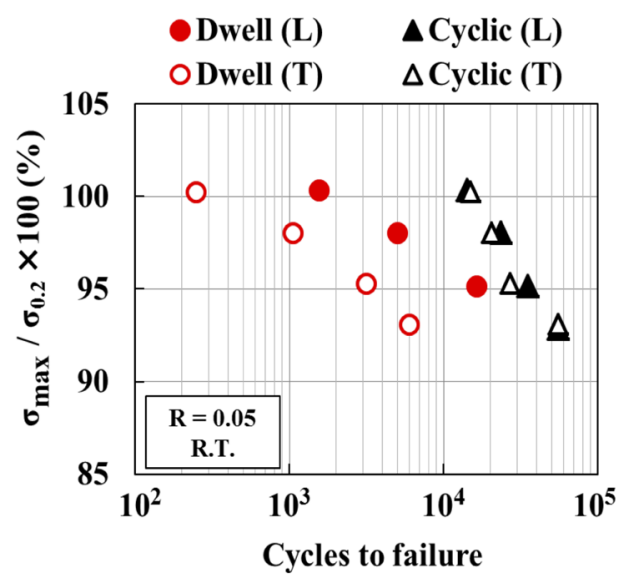

Figure 2. Relationship between the ratio of maximum stress to $0.2 \%$ proof stress and cycles of failure.

SEM images of the fracture surface after fatigue tests at the stress ratio of $95 \%$ are shown in Figure 3 . In cyclic fatigue loaded in the $\mathrm{T}$ direction and dwell fatigue loaded in the $\mathrm{L}$ direction, small facets, which size were about an alpha grain, were observed at the initiation site near the surface. These fracture surface morphologies are common, changing from striation to dimple towards the center of the specimens. On the other hand, in dwell fatigue loaded in the T direction, a large facet of 200 to $300 \mu \mathrm{m}$, which was presumed to be the initiation site, were observed. Detailed examinations of the large facet (Figure 4) revealed that the large facets consisted of propagation facets with ridges like river pattern, and initiation facets with less pattern. In addition, it was observed that cracks propagated from multiple initiation facets. Analysis of the propagation facets using EBSD method revealed that the propagation facets were approximately parallel to (0001) $\alpha$. As the crack propagated to the inside of the test piece, the fracture surface morphology shifted to the dimple morphologies. The fracture surface morphology was similar, but a little striation was observed beside the large facet at the stress ratio of $93 \%$. 

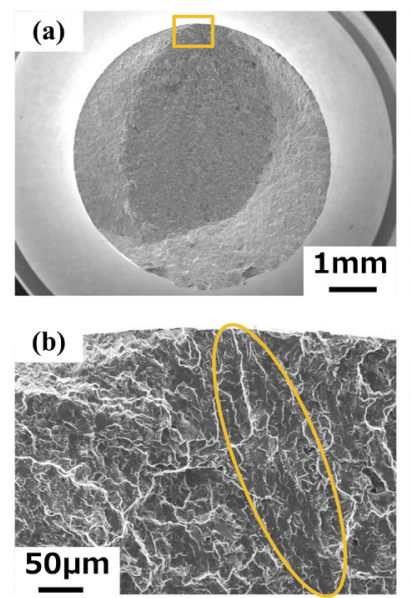

(c)

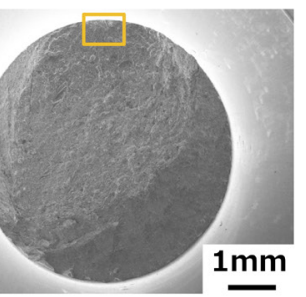

(d)

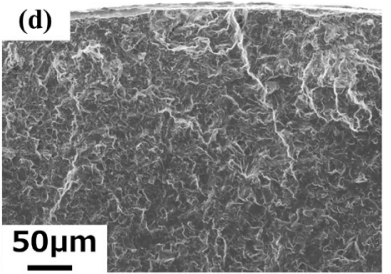

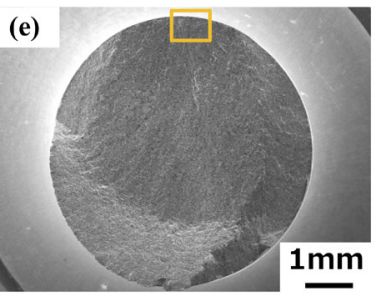

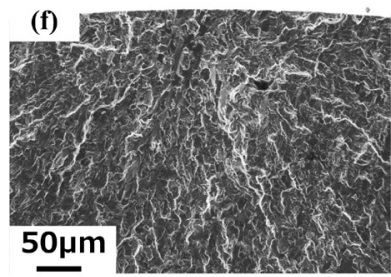

Figure 3. SEM images of fracture surface for (a)(b) dwell fatigue (T) at $\sigma_{\max }=95 \% \times \sigma_{0.2}(870 \mathrm{MPa})$, (c)(d) dwell fatigue (L) at $95 \% \times \sigma_{0.2}(825 \mathrm{MPa})$, (e)(f) cyclic fatigue (T) at $\sigma_{\max }=95 \% \times \sigma_{0.2}(870 \mathrm{MPa})$. (b) A large facet can be observed.

(a)

Initiation facet

$\rightarrow$ Propagation facet

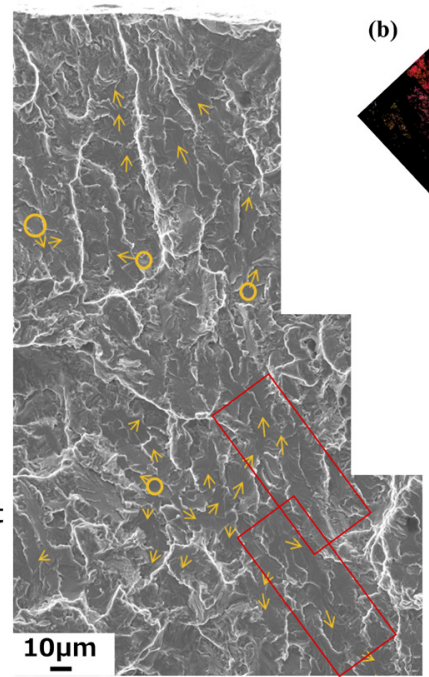

(b)

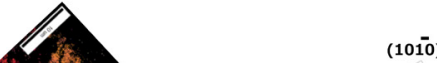

(1010)
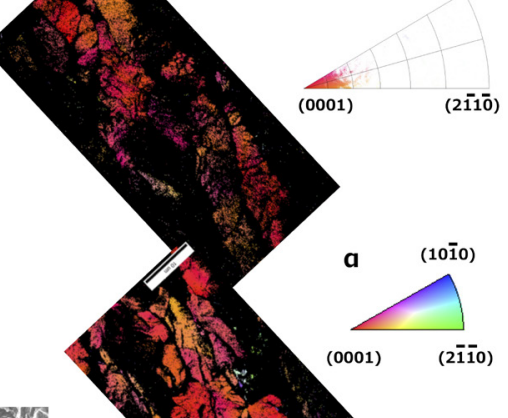

(1)

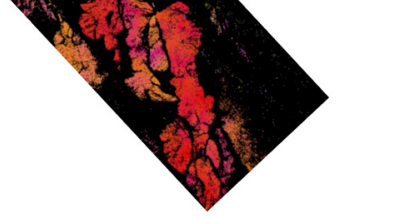

Figure 4. (a) SEM image and (b) inverse pole figure maps of the fracture surface for dwell fatigue (T) at $\sigma_{\max }=95 \% \times \sigma_{0.2}(870 \mathrm{MPa})$.
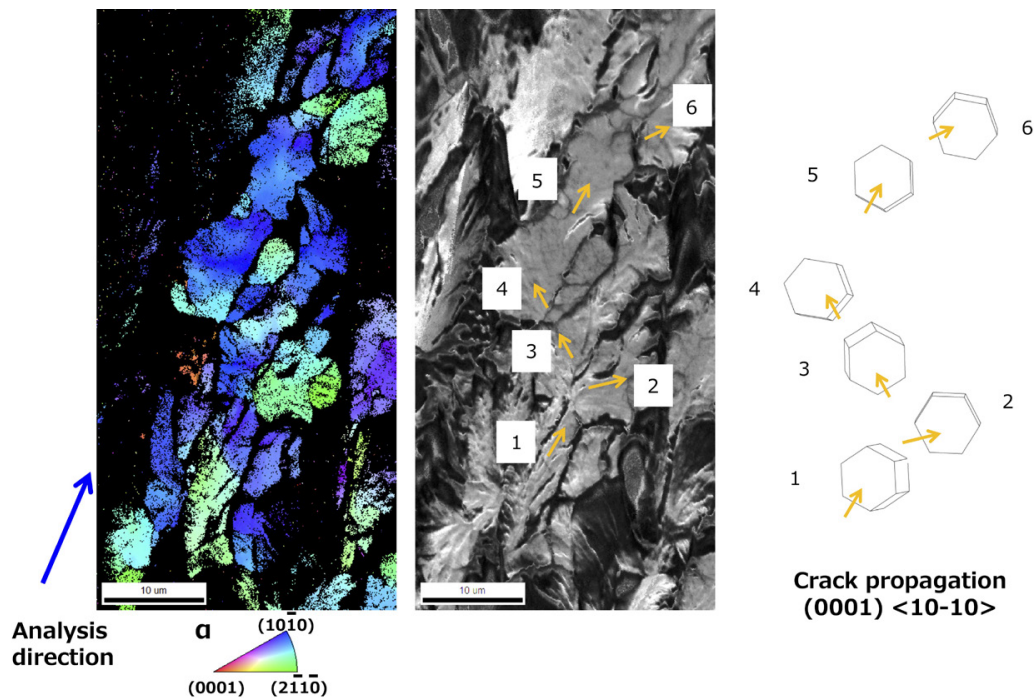

Figure 5. (a) Inverse pole figure map and (b) image quality map of the fracture surface for dwell fatigue (T) at $\sigma_{\mathrm{max}}=95 \% \times \sigma_{0.2}(870 \mathrm{MPa})$. 
Figure 5 shows the results of further detailed analysis of the propagation facets shown in Figure 4. Here, the crystal orientation analysis was based on the direction in which the crystal grains were macroscopically elongated. The direction was generally parallel to $\langle 10-10\rangle_{\alpha}$, which likely corresponds to the axial direction of the bar. The crack propagation direction appeared to correspond to $\langle 10-10\rangle_{\alpha}$, including the case if the crack propagation direction changed.

The large facets, which must be formed by the combination of microtexture and load shedding, would affect the dwell fatigue life. Bowen reported that $(0001)<10-10>$ (TL) orientation is lower static fracture toughness [11] and harder to observe striation [12] as compared to other orientations. There would be a possibility that the results have some relations to the tendency of the crack growth easiness in the $(0001)<10-10>$ direction.

Figure 6 shows SEM images of the fracture surface after dwell fatigue tests and creep tests at the stress ratio of $100 \%$. In dwell fatigue test for L direction, it was observed that almost the entire surface were dimple morphology and some regions were fine facets with a size of alpha grain diameter. As for $\mathrm{T}$ direction in dwell fatigue, the facet size was larger up to about $100 \mu \mathrm{m}$ in length. It is considered that the multiple voids which occurred simultaneously grew and coalesced, resulting fracture. The fracture surface morphology after creep tests were similar to that for the dwell fatigue test at the stress ration of $100 \%$.

Figure 7 shows the summary of the relationship between the fracture surface morphology and stress ratio. The difference in fracture surface morphology are shown clearly. At the stress ratio of $95 \%$, common fatigue fracture surface morphology is formed for dwell fatigue in $\mathrm{L}$ direction and for cyclic fatigue in both $\mathrm{L}$ and $\mathrm{T}$ direction. On the other hand, large facets are formed for dwell fatigue in $\mathrm{T}$ direction. As the stress increased, the facet size decreased and changed to the dimple morphology including small facets. At higher stress ratio, the morphology of fracture surface in dwell fatigue resembles to that of the creep. It is considered that the facet size is affected by stress ratio and is restricted by the size of MTR with specific orientation.
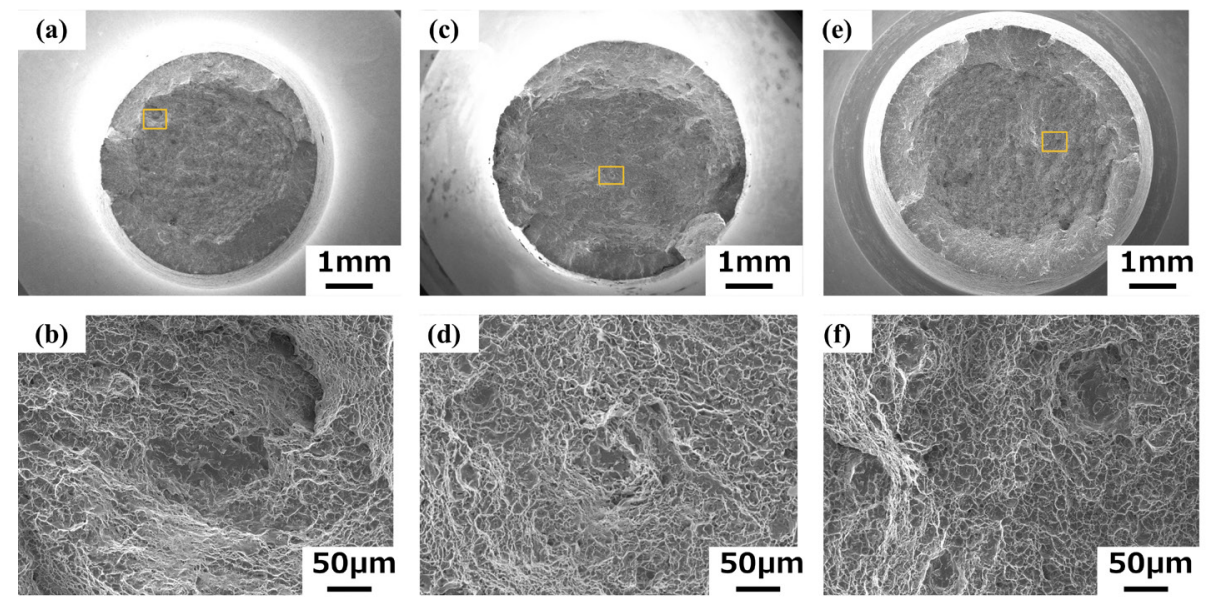

Figure 6. SEM images of fracture surfaces for (a)(b) dwell fatigue (T) at $\sigma_{\max }=100 \% \times \sigma_{0.2}(915 \mathrm{MPa})$, (c)(d) dwell fatigue (L) at $\sigma_{\mathrm{max}}=$ $100 \% \times \sigma_{0.2}(870 \mathrm{MPa}),(\mathrm{e})(\mathrm{f})$ creep $(\mathrm{T})$ at $\sigma=100 \% \times \sigma_{0.2}(915 \mathrm{MPa})$.

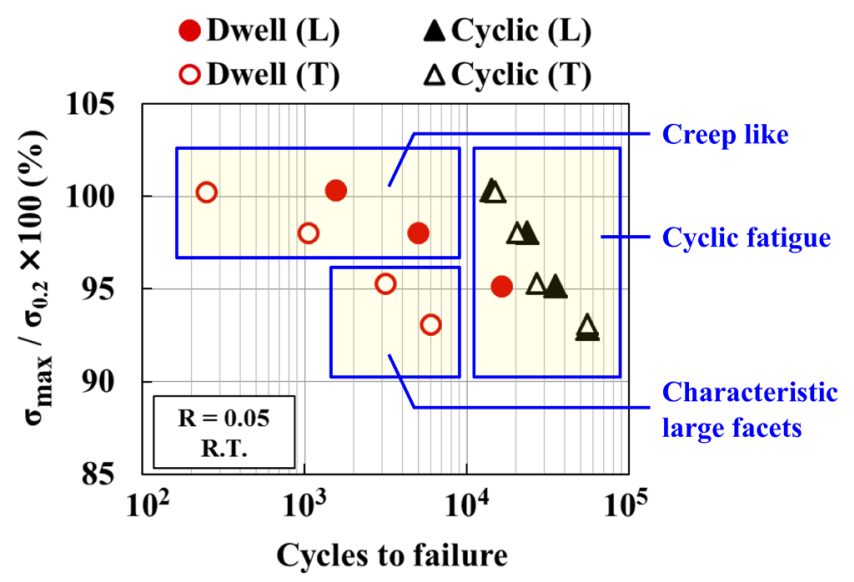

Figure 7. Relationship between S-N curves and fracture surface morphologies for fatigue tests. 


\section{(2) Strain behavior analysis}

Figure 8 shows the relationship between the strain rate at the half of life time and the rupture time in creep and dwell fatigue tests. Here, the strain rate in the dwell fatigue test is determined from the strain change at the end of the holding time of each cycle and the holding time, and the rupture time is defined by the accumulated holing time. The results of creep test are expressed by Monkman-Grant relationship. Although, in the case of dwell fatigue, the same relationship can be observed when the stress ratio is larger than $95 \%$, but the relationship shifts downwards when the stress ratio is $95 \%$ or less. The main reason for the transition is considered to be the change in the damage behavior. The fatigue crack causes the locally concentrated damage and decreases macroscopic plastic strain, while the uniform ductile fracture occurs generally in creep deformation. As shown in Figure 8, the strain rate is considerably higher for dwell fatigue than for creep when compared in the same stress and loading direction. In dwell fatigue, both work hardening and cyclic softening occurs, so if the accumulated dislocation structure is relaxed and stabilized, it would cause the acceleration of strain rate by decreasing internal stress. The effect of cyclic softening and dislocation structure should be investigated in further work.

Then, it is focused in the plastic strain range in the hysteresis loop of stress-strain curve at the half of life cycles. Figure 9 shows the relationship between the inelastic strain range and the cycles to failure. Here, the inelastic strain range, which includes plastic strain and creep strain during holding time, was measured at the mean stress. It is obvious that the inelastic stress ranges for dwell fatigue are much higher than for cyclic fatigue, and that indicates the fast damage accumulation in dwell fatigue. It is also obvious that the relationships for cyclic fatigue and for dwell fatigue are represented by different inclinations. The reason would be that the increasing strain in each cycle by tensile stress, so called ratchet strain, accelerates the decrease in dwell fatigue life. As the maximum stress increases further, the effect of creep increases and fatigue life decreases. The difference in loading direction is considered to be caused by the difference in strain concentration due to the effect of microtexture, that is, most grains correspond to soft grain in the L direction, but soft grain ratio is lower in the $\mathrm{T}$ direction.

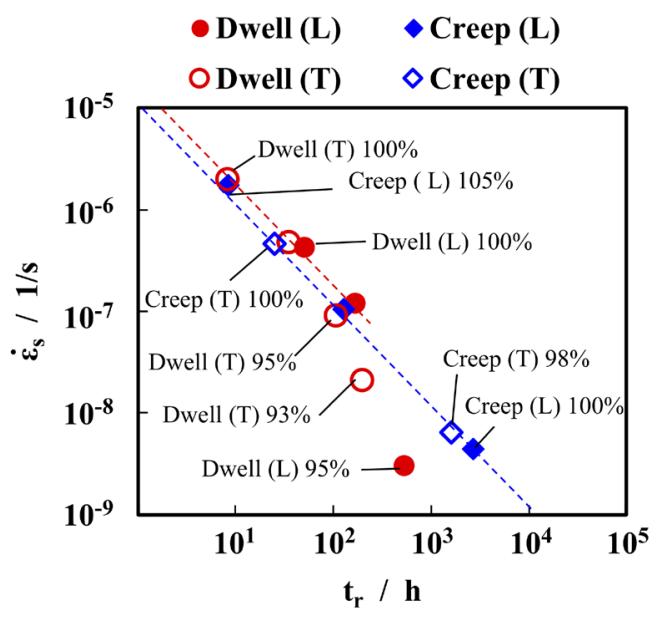

Figure 8. Relationship between strain rate at the half of the life time and time to rupture.

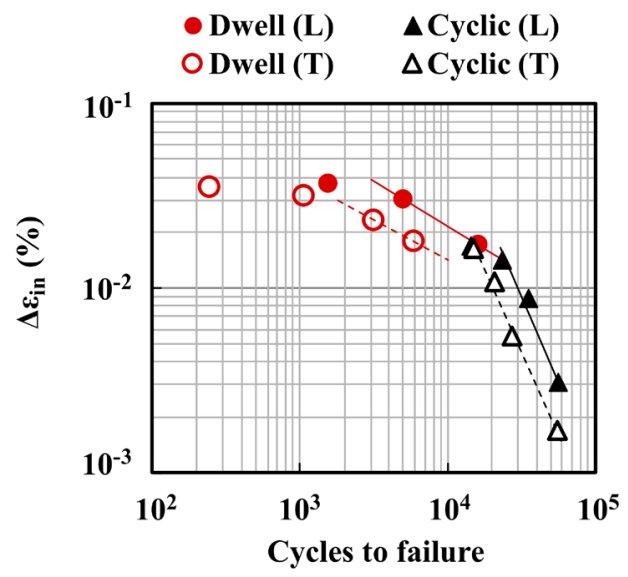

Figure 9. Relationship between inelastic strain rage $\left(\Delta \varepsilon_{\mathrm{in}}\right.$, plastic strain range including creep strain) at the half of the fatigue life and cycles to failure. 
The results of the dwell fatigue at stress ratio of $93-95 \%$ in $\mathrm{T}$ direction appear to deviate from both creep and cyclic fatigue behavior, as shown in Figure 8 and 9. The deviation suggests that the transition or mixture of fracture behavior occurs in these conditions. The fracture behavior around the transition region should be clarified quantitatively to predict dwell fatigue life.

\section{Conclusions}

Dwell fatigue properties and fracture surface characteristics of Ti-6Al-4V forged round bar with highly oriented texture were investigated by loading longitudinal $(\mathrm{L})$ and transverse $(\mathrm{T})$ direction of the bar. The results can be summarized as follows.

(1) The dwell debits are 2.1 in the $\mathrm{L}$ direction and 8.6 in the $\mathrm{T}$ direction when the maximum stress is $95 \%$ of $\sigma_{0.2}$.

(2) When the maximum stress is $95 \%$ of $\sigma_{0.2}$ in the $\mathrm{L}$ direction, crack initiates near the surface, then propagates inward forming striations and dimples like cyclic fatigue. In contrast, when the maximum stress is 93 to $95 \%$ of $\sigma_{0.2}$ in the T direction, crack initiates at the subsurface, then propagates forming characteristic large facets.

(3) When the maximum stress exceeds $95 \%$ of $\sigma_{0.2}$, regardless of $\mathrm{L}$ direction or $\mathrm{T}$ direction, the fracture surface becomes almost the same as that of room temperature creep.

(4) The relationship between strain rate and time to rupture in dwell fatigue follows to that in creep at the maximum stress higher than $95 \%$ of $\sigma_{0.2}$, and deviates at lower maximum stress. The transition corresponds to the change in the fracture surface morphology.

\section{$\underline{\text { References }}$}

[1] Bache, M. R. : International journal of fatigue 25.9-11 (2003) 1079-1087

[2] Pilchak, Adam L., Wei Jie Lu, and James C. Williams. : Mater Sci Forum Vol.710 (2012) 85-92

[3] Evans WJ, Bache MR : Int J Fatigue 16 (1994) 443-52.

[4] Hasija, V., Ghosh, S., Mills, M. J., Joseph, D. S. : Acta Mater. 51 (2003) 4533-4549

[5] Pilchak, A. L., and J. C. Williams. : Metallurgical and Materials Transactions A 42.4 (2011) 1000-1027

[6] Pilchak, Adam L. : Scripta Materialia 68.5 (2013) 277-280

[7] Lang, M. : Acta materialia 47.11 (1999) 3247-3261

[8] Mori, K. et al.: CAMP-ISIJ Vol.31 (2018) 397( in Japanese)

[9] Mori, K. et al.: CAMP-ISIJ Vol.31 (2018) 926 (in Japanese)

[10] Hashimoto, S. et.al. : CAMP-ISIJ Vol.312 (2019) 378 (in Japanese)

[11]Bowen, A.W. : Acta Metallugica Vol.26(1978) 1423-1433

[12]Bowen, A.W. : Acta Metallugica Vol.23(1975) 1401-1409 